\title{
Suitability of Data for the Surveillance of Toxicological Events in Companion Animals
}

\author{
Alexandra Swirski*, David Pearl, Olaf Berke, Terri O’Sullivan and Deborah Stacey
}

Population Medicine, University of Guelph, N1H7J6, ON, Canada

\section{Objective}

Our objective was to assess the suitability of the data collected by the Animal Poison Control Center, run by the American Society for the Prevention of Cruelty to Animals, for the surveillance of toxicological exposures in companion animals in the United States.

\section{Introduction}

There have been a number of non-infectious intoxication outbreaks reported in North American companion animal populations over the last decade ${ }^{1}$. The most devastating outbreak to date was the 2007 melamine pet food contamination incident which affected thousands of pet dogs and cats across North America ${ }^{1}$. Despite these events, there have been limited efforts to conduct real-time surveillance of toxicological exposures in companion animals nationally, and there is no central registry for the reporting of toxicological events in companion animals in the United States. However, there are a number of poison control centers in the US that collect extensive data on toxicological exposures in companion animals, one of which is the Animal Poison Control Center (APCC) operated by the American Society for the Prevention of Cruelty to Animals (ASPCA). Each year the APCC receives thousands of reports of suspected animal poisonings and collects extensive information from each case, including location of caller, exposure history, diagnostic findings, and outcome. The records from each case are subsequently entered and stored in the AnTox database, an electronic medical record database maintained by the APCC. Therefore, the AnTox database represents a novel source of data for real-time surveillance of toxicological events in companion animals, and may be used for surveillance of pet food and environmental contamination events that may negatively impact both veterinary and human health.

\section{Methods}

Recorded data from calls to the APPC were collected from the AnTox database from January 1, 2005 to December 31, 2014, inclusive. Sociodemographic data were extracted from the American 2010 decennial census and the American Community Surveys. Choropleth maps were used for preliminary analyses to examine the distribution of reporting to the hotline at the county-level and identify any "holes" in surveillance. To further identify if gaps in reporting were randomly distributed or tended to occur in clusters, as well as to look for any predictable spatial clusters of high rates of reporting, spatial scan statistics, based on a Poisson model, were employed. We fitted multilevel logistic regression models, to account for clustering within county and state, to identify factors (e.g., season, human demographic factors) that are related to predictable changes in call volume or reporting, which may bias the results of quantitative methods for aberration/outbreak detection.

\section{Results}

Throughout the study period, over $40 \%$ of counties reported at least one call to the hotline each year, with the majority of calls coming from the Northeast. Conversely, there was a large "hole" in coverage in Midwestern and southeastern states. The location of the most likely high and low call rate clusters were relatively stable throughout the study period and were associated with socioeconomic status (SES), as the most likely high risk clusters were identified in areas of high SES. Similar results were identified using multivariable analysis as indicators of high SES were found to be positively associated with rates of calls to the hotline at the county-level.

\section{Conclusions}

Socioeconomic status is a major factor impacting the reporting of toxicological events to the APCC, and needs to be accounted for when applying cluster detection methods to identify outbreaks of mass poisoning events. Large spatial gaps in the network of potential callers to the center also need to be recognized when interpreting the spatiotemporal results of analyses involving these data, particularly when statistical methods that are highly influenced by edge effects are used.

\section{Keywords}

toxicology; companion animals; multilevel models; geospatial analysis

\section{Acknowledgments}

The authors would like to thank the ASPCA for providing the data for the project.

\section{References}

1. Brown CA, Jeong K-S, Poppenga RH, et al. Outbreaks of Renal Failure Associated with Melamine and Cyanuric Acid in Dogs and Cats in 2004 and 2007. J Vet Diagnostic Investig 2007;19:525-531.

\author{
*Alexandra Swirski \\ E-mail: aswirski@uoguelph.ca
}

